\title{
Direct measurement of concurrence for atomic two-qubit pure states
}

\author{
G. Romero ${ }^{1}$, C.E. López ${ }^{1}$, F. Lastra ${ }^{1}$, E. Solano ${ }^{2,3}$, and J.C. Retamal ${ }^{1}$ \\ ${ }^{1}$ Departamento de Física, Universidad de Santiago de Chile, Casilla 307 Correo 2, Santiago, Chile \\ ${ }^{2}$ Physics Department, ASC, and CeNS, Ludwig-Maximilians-Universität, Theresienstrasse 37, 80333 Munich, Germany \\ ${ }^{3}$ Sección Física, Departamento de Ciencias, Pontificia Universidad Católica del Perú, Apartado 1761, Lima, Peru
}

(Dated: July 18, 2018)

\begin{abstract}
We propose a general scheme to measure the concurrence of an arbitrary two-qubit pure state in atomic systems. The protocol is based on one- and two-qubit operations acting on two available copies of the bipartite system, and followed by a global qubit readout. We show that it is possible to encode the concurrence in the probability of finding all atomic qubits in the ground state. Two possible scenarios are considered: atoms crossing 3D microwave cavities and trapped ion systems.
\end{abstract}

PACS numbers: $03.67 . \mathrm{Mn}, 42.50 . \mathrm{Ct}, 42.50 . \mathrm{Vk}$

Quantum entanglement is a key resource for quantum information and quantum computation [1]. This intriguing property lies at the heart of the EinsteinPodolsky-Rosen paradox [2]. Entangled states have been implemented in different physical setups, for example, in photons [3], massive particles like trapped ions [4], nuclear magnetic resonance [5], atoms in cavities 6], quantum dots 7], among others. On the other hand, the quantification of the degree of entanglement for an arbitrary number of qubits is still an open problem in quantum information [8]. Arguably, the most valuable entanglement measure is the entanglement of formation (EOF) [9], which quantifies the minimal cost needed to prepare a certain quantum state in terms of EPR pairs. Many efforts have been devoted to the derivation of the EOF through analytical and numerical approaches. In an important contribution it has been shown that $\mathrm{EOF} E_{f}(\rho)$ for an arbitrary two-qubit state $\rho$ can be defined in terms of an exactly calculable quantity: the concurrence $C$ [10]. This quantity can be defined as $C(\rho)=\max \left\{0, \lambda_{1}-\lambda_{2}-\lambda_{3}-\lambda_{4}\right\}$, where the $\lambda_{i}$ 's are square roots in decreasing order of the eigenvalues of matrix $\rho \tilde{\rho}$ with $\tilde{\rho}=\sigma_{y} \otimes \sigma_{y} \rho^{*} \sigma_{y} \otimes \sigma_{y}, \sigma_{y}$ being the usual Pauli operator. Remarkably, for a pure state this concurrence is reduced to the simple expression

$$
C\left(\left|\psi_{i}\right\rangle\right)=\left|\left\langle\psi\left|\sigma_{y} \otimes \sigma_{y}\right| \psi^{*}\right\rangle\right| .
$$

A straightforward method for measuring entanglement would be a complete tomographic reconstruction of the quantum state 11]. In this case, the reconstruction of a two-qubit state requires the readout of 15 parameters. Additionally, theoretical proposals based on entanglement Witness operator 12], positive maps [13], and two-particle interference [14], have been introduced. Recently, the direct measurement of concurrence of a twophoton entangled state was implemented in the lab [15]. This experiment is based on the fact that the concurrence information of a two-qubit pure state is encoded in the probability of observing the two copies of the first subsystem in an antisymmetric state [16]. Without any doubt, it would be desirable to translate these ideas to the case of matter qubits where diverse physical setups have reached high level of quantum control.

In this work, we propose a method to measure the concurrence of a two-qubit pure state in matter qubits. The proposed technique relies on the availability of two copies of the bipartite state and the direct measurement of the occupation probability of the collective state of both copies. We illustrate this protocol with two examples, Rydberg atoms crossing 3D microwave cavities [6] and confined ions in a linear Paul trap [4].

The central idea of this proposal is the transformation of the separable state of two copies into a state where the value of the concurrence will be loaded in the probability amplitude to have all the qubits in the ground state. The required operations are $\sigma_{y}$ unitaries and local rotations $R$, as well as a controlled-not gate (CNOT), followed by a global measurement of all four qubits. In Fig. 1 we present a quantum circuit describing the proposed protocol. Here, the first two channels stand for the entangled state we want to measure, the third and fourth channel denote the copy of the two-qubit state. Finally, the measurement is produced through the detection of all qubits in the ground state.

Let us assume that we want to measure the concur-

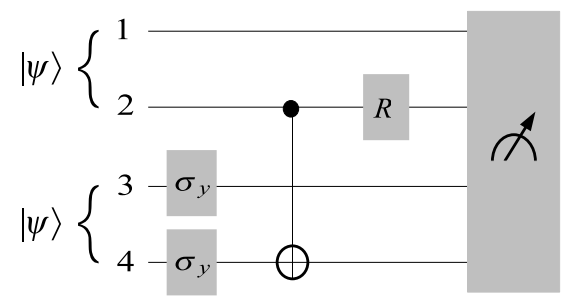

FIG. 1: Quantum circuit describing a direct measurement of the concurrence of a two-qubit pure state, where two copies are available. It involves a controlled-not gate, as well as $\sigma_{y}$ unitaries and other simple $R$ qubit rotations, followed by the joint measurement of the four qubits. 
rence of the general two-qubit pure state

$$
|\psi\rangle=c_{0}|g g\rangle+c_{1}|g e\rangle+c_{2}|e g\rangle+c_{3}|e e\rangle,
$$

and we are provided with two decoupled copies of it $|\psi\rangle \otimes|\psi\rangle$. It can be shown from Eq. (10) that the concurrence of state $|\psi\rangle$ in terms of coefficients $c_{i}$ is given by $C(|\psi\rangle)=2\left|c_{1} c_{2}-c_{0} c_{3}\right|$. Following the suggested quantum circuit of Fig. 1] we apply local operations on the second copy such that the global state is described by $|\Phi\rangle=|\psi\rangle \otimes\left(\sigma_{y} \otimes \sigma_{y}|\psi\rangle\right)$. This state can be written as a superposition of states having a number excitations $k$ ranging from 0 to 4 ,

$$
\begin{aligned}
|\Phi\rangle= & -c_{0} c_{3}|g g g g\rangle \\
& \left.\left.+c_{2} c_{0} \mid \text { ggge }\right\rangle+c_{0} c_{1} \mid \text { gge }\right\rangle \\
& \left.\left.-c_{1} c_{3} \mid \text { gegg }\right\rangle-c_{2} c_{3} \mid \text { eggg }\right\rangle \\
& \left.\left.\left.-c_{0}^{2} \mid \text { ggee }\right\rangle-c_{3}^{2} \mid \text { eegg }\right\rangle+c_{1} c_{2} \mid \text { gege }\right\rangle \\
& \left.\left.\left.+c_{1}^{2} \mid \text { geeg }\right\rangle+c_{2}^{2} \mid \text { egge }\right\rangle+c_{2} c_{1} \mid \text { egeg }\right\rangle \\
& \left.\left.-c_{1} c_{0} \mid \text { geee }\right\rangle-c_{2} c_{0} \mid \text { egee }\right\rangle \\
& \left.\left.+c_{3} c_{2} \mid \text { eege }\right\rangle+c_{3} c_{1} \mid \text { eeeg }\right\rangle \\
& \left.-c_{3} c_{0} \mid \text { eeee }\right\rangle .
\end{aligned}
$$

Now, we apply a CNOT operation between the second qubit acting as the control and the fourth qubit acting as the target, followed by a rotation on the second qubit. The CNOT gate in this protocol is defined such that if the control qubit is in state $|g\rangle$ the target is not affected, conversely, if the control is in the state $|e\rangle$ the target is flipped. The subsequent rotation $R_{2}^{-}$acting on qubit 2 can be taken from $R_{j}^{ \pm}:|g\rangle_{j} \rightarrow\left(|g\rangle_{j} \pm|e\rangle_{j}\right) / \sqrt{2}$ and $|e\rangle_{j} \rightarrow\left(|e\rangle_{j} \mp|g\rangle_{j}\right) / \sqrt{2}$. After the CNOT and $R_{2}^{-}$operations, the state of the overall system becomes

$$
\begin{aligned}
\left|\Phi_{1}\right\rangle=\frac{1}{\sqrt{2}} & \left.\left\{A_{-} \mid \text {gggg }\right\rangle+A_{+} \mid \text {gegg }\right\rangle \\
& \left.\left.+B_{-} \mid \text {ggge }\right\rangle-B_{+} \mid \text {gege }\right\rangle \\
& \left.\left.+2 c_{2} c_{3} \mid \text { eegg }\right\rangle-2 c_{0} c_{1} \mid \text { geeg }\right\rangle \\
& \left.\left.+C_{10}^{-} \mid \text {ggee }\right\rangle+C_{10}^{+} \mid \text {geee }\right\rangle \\
& \left.\left.+C_{23}^{-} \mid \text {egge }\right\rangle-C_{23}^{+} \mid \text {eege }\right\rangle \\
& \left.\left.+A_{-} \mid \text {egeg }\right\rangle-A_{+} \mid \text {eeeg }\right\rangle \\
& \left.\left.\left.+B_{+} \mid \text {eeee }\right\rangle-B_{-} \mid \text {egee }\right\rangle\right\}
\end{aligned}
$$

where $A_{ \pm}=c_{1} c_{2} \pm c_{0} c_{3}, B_{ \pm}=c_{0} c_{2} \pm c_{1} c_{3}$, and $C_{i j}^{ \pm}=$ $c_{i}^{2} \pm c_{j}^{2}$. We observe that in Eq. (4) the concurrence information of state $|\psi\rangle$ is present in the coefficient $A_{-}$ through

$$
C(|\psi\rangle)=2 \sqrt{2 P_{\text {gggg }}}
$$

where $P_{g g g g}=\left|A_{-}\right|^{2} / 2$. Clearly, a similar argumentation leads also to $C(|\psi\rangle)=2 \sqrt{2 P_{\text {egeg }}}$. We will clarify our choice below when discussing applications to specific experimental setups.

We consider now the proposed protocol for the case of atoms flying through 3D microwave cavities, an important physical setup where fundamental tests of quantum mechanics have been realized [6].

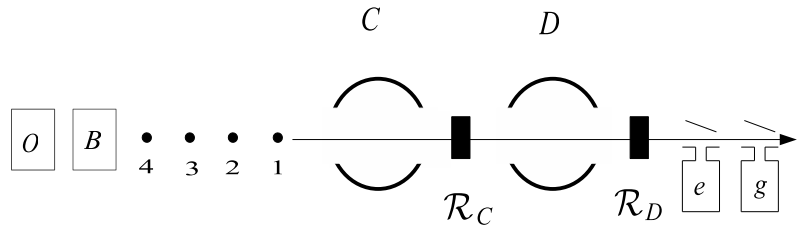

FIG. 2: Protocol for measuring concurrence in microwave 3D cavity QED using two cavities and two Ramsey regions.

The proposed protocol will make use of two cavities, two Ramsey regions, and Rydberg atoms crossing them at given velocities, see Fig. 2 It relies on present efforts to develop two-cavity setups [17], but see also other multi-cavity projects [18, 19]. The first cavity $C$ is used to create two copies of the same entangled two-atom state in a consecutive manner. We make use of an entangling technique that has already been experimentally demonstrated in Ref. 20], following the proposal of Ref. [21]. Along these lines we are entitled to say that a general entangled state of the form $\alpha|g e\rangle+\beta|e g\rangle$ could be produced in the lab. In Ref. [20], two Rydberg atoms, with a relative delay $\tau$, are sent from $B$ with velocities $v$ and $w(w>v)$ such that they cross inside the cavity, determining in this way the desired effective Rabi angle. We propose here to create the two required copies one after the other, where the atoms of each pair will have the same velocities $v$ and $w$, and a suitable delay time $\tau^{\prime}$ between atoms 2 and 3 . We will see below that while requiring the atom pairs $\{1,2\}$ and $\{3,4\}$ to cross inside cavity $C$, for generating the same entangled state $|\Psi\rangle$, atoms 2 and 4 will not need to cross in $D$ to produce the CNOT gate.

Before cavity $C$, see Fig. 2 the four atoms follow the natural order $\{4,3,2,1\}$, from left to right. Immediately after cavity $C$, the four atoms encoding the initial state $|\Psi\rangle \otimes|\Psi\rangle$, follow the ordering $\{3,4,1,2\}$ due to the timing and velocities mentioned above. To begin with the protocol described in Fig. 1 we allow now, atoms 4 and 3 to cross the Ramsey region where local unitaries $\sigma_{y}$ are applied. We consider that Ramsey regions were not active when atoms 2 and 1 passed through at an earlier time. We recall that Ramsey zones, implementing different local rotations are well-controlled and accurate devices, representing an important building block of present technology in 3D microwave cavities [6]. Note that short before entrying cavity $D$, it would be preferable to have the following ordering $\{3,1,4,2\}$. This exchange of positions between atoms 1 and 4 could be easily achieved by proper tuning of parameters $v, w, \tau, \tau^{\prime}$, and the distance between cavities.

The second step of the protocol is the implementation of a $\operatorname{CNOT}(2,4)$ gate between control atomic qubit 2 and target atomic qubit 4 . As explained before, atom 2 arrives first to cavity $D$ followed by atom 4 . It can be easily proved that this gate is equivalent to the successive oper- 


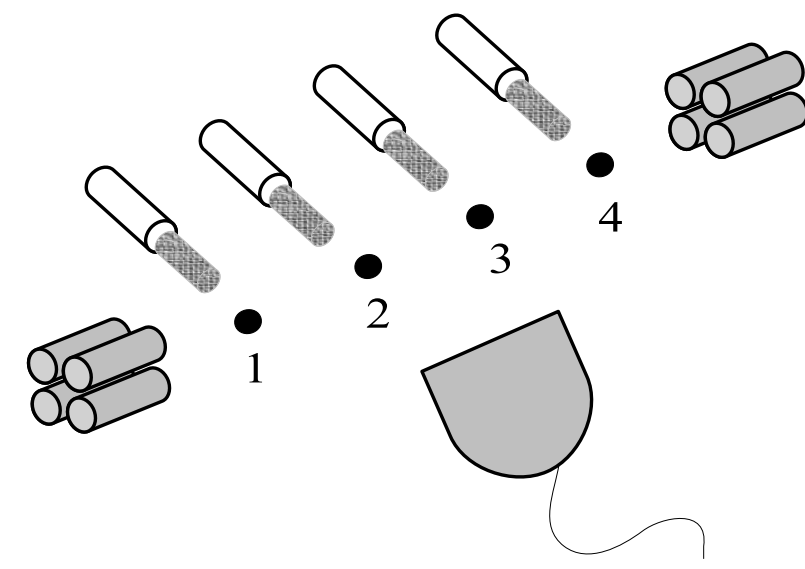

FIG. 3: Four ions in a linear Paul trap that can be individually addressed, measured, and coupled to a collective motional degree of freedom to implement the protocol of Fig. [1]

ations $R_{4}^{+} \times \operatorname{CPHASE}(2,4) \times R_{4}^{-}$. The $\operatorname{CPHASE}(2,4)$ gate acts as follows: $|e\rangle_{2}|e\rangle_{4} \rightarrow-|e\rangle_{2}|e\rangle_{4}$, while the other basis states, $\left\{|g\rangle_{2}|g\rangle_{4},|g\rangle_{2}|e\rangle_{4},|e\rangle_{2}|g\rangle_{4}\right\}$, remain unchanged. To achieve this goal we map first the qubit of atom 2 onto the photonic state of cavity $D$. Then, atomic qubit 4 is transformed due to $R_{4}^{-}$and enters into cavity $D$ to perform a CPHASE with the photonic qubit, that is, $|e\rangle|1\rangle \rightarrow-|e\rangle|1\rangle$, leaving other states unchanged. We suggest the use of the CPHASE gate implemented experimentally in Ref. 22]. Along these lines, we propose the use of an upper auxiliary level $|i\rangle$ allowing a $2 \pi$-pulse rotation in the subspace $\{|e\rangle|1\rangle,|i\rangle|0\rangle\}[23]$. Finally, atomic qubit 4 is rotated through the action of $R_{4}^{+}$, while the photonic qubit is mapped back onto an additional atom 5 in its ground state.

As is evident from above, atom 2 is lost in this process but its logical information is carried now by atom 5. A last step consists on measuring the level statistics of all qubits after a final rotation $R_{5}^{-}$is implemented on atomic qubit 5, following the protocol of Fig. 1 As shown in Eq. (5), the probability of finding all relevant atoms $\{5,3,1,4\}$ in the ground state will provide us with a valuable information: the concurrence of the entangled pure state $|\Psi\rangle$. Clearly, following Eq. (4), we can obtain similar information if we use the probability $P_{\text {egeg }}$.

There are additional technical points in order. First, it would be desirable that atom 5 is sent with the proper timing and velocity so that it can retrieve the photonic qubit of cavity $D$ before decoherence destroys the encoded information of atom 2. Second, the proper tuning of the relative frequency of cavities $C$ and $D$ can only be decided when all experimental parameters, including inter-cavity distance and atomic transitions, are decided. Third, we rely our proposal on the possibility of switching on and off at any desired time the Ramsey regions, as well as in controlled DC-induced shifts in the atomic transition frequencies [24].
Alternatively, the protocol of Fig. 1 could be implemented straightforwardly in four trapped ions, see Fig. [3. as will be discussed below. For achieving that goal we require to implement $\sigma_{y}$ unitaries, local rotations $R^{ \pm}$, and a CNOT gate, all of which have already been implemented in the lab with high precision in several trapped ions. That is, we rely on the possibility of implementing individual addressing on each of the four ions, for the sake of individual control and readout. Typically, the measurement of each ion is done by means of an electron-shelving technique, where an internal level, say $|e\rangle$, is coupled to an auxiliary level $|c\rangle$ that decays cyclicly back to $|e\rangle$. The abundance of fluorescence photons implies the projection of the qubit on state $|e\rangle$, and the absence of fluorescence photons warrants the projection of the qubit on the other state $|g\rangle$. However, we remark that, for measuring the concurrence according to the proposed protocol, it is not necessary to realize an individual readout of the ionic qubits. We propose here the use of a technique that may be called global electron-shelving [25], where the required measurement of $P_{g g g g}$ of Eq. (5) is reduced to a singlebit yes/no question. Given that all ions are identical, we can apply the same electron-shelving laser pulse globally and simultaneously, but each ion will perform its individual associated cyclic transition. Only the absence of fluorescence photons warrants the projection of the fourqubit state onto state $|g\rangle|g\rangle|g\rangle|g\rangle$, while the presence of fluorescence photons implies a projection on any other four-qubit state. It may even happen that, while implementing the yes/no global photon statistics, the multi-ion case produces a higher fidelity in the desired probability measurement when compared to the individual readout case. In this manner we would accomplish the measurement of the concurrence through a simplified and global readout scheme for $P_{g g g g}$ of Eq. (5).

In conclusion, we have presented a realistic protocol for measuring directly the concurrence of a two-qubit pure state in matter qubits, as long as two copies and a few simple operations are available. We have shown that it can be applied in a two-cavity setup in microwave 3D cavity QED and straightforwardly in trapped ion systems. We believe that this proposal could be implemented with present technology and will boost research in the hard task of quantifying entanglement of small dimensional systems.

The authors thanks T. Schätz for useful discussions. GR acknowledges financial support from CONICYT Ph.D. Programm Fellowships, CEL and FL from MECESUP USA0108, JCR from Fondecyt 1030189 and Milenio ICM P02-049, and ES from EU RESQ, EuroSQIP, and DFG SFB 631 projects. CEL thanks also DIGEGRA USACH and Jan von Delft for hospitality at LMU. 
[1] M.A. Nielsen and I.L. Chuang, Quantum Computation and Quantum Information (Cambridge Univ. Press., Cambridge, 2000).

[2] A. Einstein, B. Podolski, and N. Rosen, Phys. Rev. 47, 777 (1935).

[3] W. Tittel and G. Weihs, Quantum Inf. Comput. 2, 3 (2001).

[4] D. Leibfried, R. Blatt, C. Monroe, and D. Wineland, Rev. Mod. Phys. 75, 281 (2003).

[5] L. M. K. Vandersypen and I. L. Chuang, Rev. Mod. Phys. 76, 1037 (2004).

[6] J.M. Raimond, M. Brune, and S. Haroche, Rev. Mod. Phys. 73, 565 (2001).

[7] R. Hanson, L.P. Kouwenhoven, J.R. Petta, S. Tarucha, L.M.K. Vandersypen, cond-mat/0610433

[8] F. Mintert, A.R.R. Carvalho, M. Kus, and A. Buchleitner, Phys. Rep. 415, 207 (2005); 419, 143 (2005).

[9] S. Hill and W. K. Wootters, Phys. Rev. Lett. 78, 5022 (1997).

[10] W. K. Wootters, Phys. Rev. Lett. 80, 2245 (1998).

[11] A.G. White, D.F.V. James, P.H. Eberhard, and P.G. Kwiat, Phys. Rev. Lett. 83, 3103 (1999).

[12] O. Gühne, P. Hyllus, D. Bruß, A. Ekert, M. Lewenstein, C. Macchiavello, and A. Sanpera, Phys. Rev. A 66, 062305 (2002).

[13] P. Horodecki and A. Ekert, Phys. Rev. Lett. 89, 127902 (2002).

[14] Jiang-Ming Cai, Zheng-Wei Zhou, and Guang-Can Guo,
Phys. Rev. A 73, 024301 (2006).

[15] S. P. Walborn, P. H. Souto Ribeiro, L. Davidovich, F. Mintert, and A. Buchleitner, Nature 440, 1020 (2006); S.J. van Enk, quant-ph/0606017 F. Mintert and A. Buchleitner, quant-ph/0605250

[16] F. Mintert, M. Kus, and A. Buchleitner, Phys. Rev. Lett. 95, 260502 (2005).

[17] S. Haroche group at École Normale Supérieure de Paris (private communication).

[18] M.F. Santos, P. Milman, L. Davidovich, and N. Zagury, Phys. Rev. A 73, 040305 (2006).

[19] P. Blythe and B. Varcoe, quant-ph/0605190

[20] S. Osnaghi, P. Bertet, A. Auffeves, P. Maioli, M. Brune, J. M. Raimond, and S. Haroche, Phys. Rev. Lett. 87, 037902 (2001).

[21] Shi-Biao Zheng and Guang-Can Guo, Phys. Rev. Lett. 85, 2392 (2000).

[22] A. Rauschenbeutel, G. Nogues, S. Osnaghi, P. Bertet, M. Brune, J. M. Raimond, and S. Haroche, Phys. Rev. Lett. 83, 5166 (2000).

[23] For pedagogical reasons we have renamed the atomic levels of Ref. 22]. For us, the lower energy level is $|g\rangle$, the intermediate one $|e\rangle$, and the upper auxiliary level $|i\rangle$.

[24] A. Rauschenbeutel, G. Nogues, S. Osnaghi, P. Bertet, M. Brune, J.-M. Raimond, and S. Haroche, Science 288, 2024 (2000).

[25] Q.A. Turchette, C.S. Wood, B.E. King, C.J. Myatt, D. Leibfried, W.M. Itano, C. Monroe, and D.J. Wineland, Phys. Rev. Lett. 81, 3631 (1998). 